'Departamento de Nutrición, Diabetes y Metabolismo.

Facultad de Medicina,

Pontificia Universidad

Católica de Chile.

2Unidad de Endocrinología y Diabetes, Departamento de Medicina Interna. Universidad de La Frontera, Temuco, Chile.

Recibido el 20 de julio de 2011, aceptado el $20 \mathrm{de}$ marzo de 2012.

Correspondencia a:

Dr. Felipe Pollak C.

Dirección: Marcoleta 354, Edificio Gastroenterología

$4^{\circ}$ piso.

Teléfono: 3543862

E-mail:fpollak@med.puc.cl

\section{Diabetes autoinmune (latente) del adulto}

\author{
FELIPE POLLAK C. ${ }^{1}$, TATIANA VÁSQUEZ A. ${ }^{2}$
}

\section{Latent autoimmune diabetes in adults}

Latent Autoimmune Diabetes in Adults (LADA) is the term used to describe adults who have a slowly progressive form of diabetes mellitus (DM) of autoimmune etiology, but that may be treated initially without insulin. Although it shares some immunological and genetic aspects with type 1 DM, it affects an age group that is typically affected by type $2 \mathrm{DM}$. Therefore, it could be considered an intermediate type. Diagnosis is based on clinical and laboratory criteria: age of onset, initial response to oral hypoglycemic agents and the presence of specific antibodies for diabetes. Although the definitive treatment is insulin, glitazones may be useful in early stages of the disease. Currently, its management represents a challenge for the physician, including specialists, and it is a form of DM to keep in mind.

(Rev Med Chile 2012; 140: 1476-1481).

Key words: Autoimmunity; Diabetes mellitus; Insulin; Thiazolidinediones.
I a diabetes mellitus (DM) tipo 1 es causada por una respuesta autoinmune que produce una destrucción progresiva de las células beta pancreáticas. Este proceso puede ser detectado por anticuerpos específicos en sangre periférica, meses o incluso años antes de la aparición clínica de la enfermedad ${ }^{1}$. Un porcentaje de las personas adultas con DM presentan estos marcadores inmunológicos, pero inicialmente no requieren insulina, por lo que la mayoría de las veces son catalogadas como diabéticos tipo 2 . Estos pacientes probablemente tengan un proceso patológico similar a aquellos con DM tipo 1, tanto por la susceptibilidad genética, la presencia de auto anticuerpos, la disminución en la secreción de insulina y la progresión hacia la insulinodependencia.

En 1986 Groop et al. informaron de un subgrupo de pacientes diabéticos que pese a tener anticuerpos específicos mostraban conservación de la función de la célula beta, con características diferentes a las clásicas DM tipo 1 y $2^{2}$. El término LADA (Latent Autoinmune Diabetes of the Adult) fue acuñado posteriormente por Tuomi et al para describir a pacientes con una forma lentamente progresiva de DM autoinmune o tipo 1 que podían ser tratados inicialmente sin insulina ${ }^{3}$. Aunque actualmente la Asociación Americana de Diabetes
(ADA) no reconoce a esta enfermedad como un tipo especifico, hay cada vez mayor información al respecto, así como grupos dedicados a su estudio.

En el norte de Europa y en América del Norte se calcula que 5 a $10 \%$ de los nuevos casos de DM en pacientes adultos podrían corresponder a esta patología ${ }^{4}$. En China, el 9,2\% de pacientes diabéticos corresponden a este tipo, con una prevalencia de $0,6 \%{ }^{5}$.

El estudio UKPDS, realizado en diabéticos tipo 2 , mostró que $10 \%$ de los pacientes presentaba anticuerpos específicos, de los cuales un número significativo de ellos podrían ser considerados como portadores de LADA ${ }^{6}$. En este estudio la presencia de anticuerpos anti decarboxilasa de ácido glutámico (anti-GAD) o anti células beta (ICA), predijo el fracaso a hipoglicemiantes orales e insulinorequirencia más temprana en el seguimiento.

\section{Fisiopatología}

La historia natural de la enfermedad no ha sido hasta ahora bien definida. Se consideran 2 posibilidades: larga historia de autoinmunidad con una lenta progresión del daño en células beta a través de los años, o bien, aparición de autoinmunidad tardía con una fase preclínica breve. 
La aparición de estas manifestaciones es el resultado de la interacción entre la susceptibilidad genética y la exposición a factores ambientales ${ }^{3}$.

\section{Genética y autoinmunidad}

Han sido descritas características similares a la DM tipo 1, como insulinitis en estudios anatomopatológicos, por lo se ha planteado que ambas enfermedades comparten aspectos fisiopatológicos. Al respecto se conoce que LADA presenta una mayor frecuencia de antígenos humanos de histocompatibilidad (HLA) característicos de DM tipo 1: HLA-DR3 (28\% de los pacientes), DR4 (27\%) y DR 3/4 (22\%), en comparación con la población general $^{7,8}$. Por otro lado, ambas enfermedades presentan anticuerpos específicos: anti-GAD, ICA, anti-tirosin fosfatasa (IA-2) y anti-insulina, siendo el primero de ellos el de mayor prevalencia, seguido por ICA ${ }^{9,10}$. Concomitantemente se ha demostrado una disminución en la frecuencia y activación de células "natural killer" en sangre periférica comparado con individuos sanos, lo que traduce un defecto en la respuesta inmune ${ }^{11}$. Estos hallazgos han sido también descritos para diabéticos tipo 1.

Finalmente, ambas presentan asociación con otras enfermedades autoinmunes, siendo la tiroiditis la más frecuente, y reportándose pacientes con anticuerpos gástricos parietales y enfermedad celíaca ${ }^{3}$.

Sin embargo, estudios recientes han descrito diferencias genéticas entre ambos tipos de DM. Andersen et al describen una mayor frecuencia de genes protectores (HLA-DQ 0602(3)/X, 0602(3)/031) y una menor de genes considerados de riesgo (HLA-DQ 0302/02, 0302/X) en comparación con pacientes diabéticos tipo 1 , tanto de aparición temprana como tardía (después de los 35 años). Según estos resultados la DM tipo 1, temprana y tardía, y el tipo LADA corresponderían a entidades diferentes dentro de un espectro de autoinmunidad donde LADA es la presentación de menor agresividad ${ }^{12}$.

\section{Factores ambientales}

Al igual que en DM tipo 1, los factores ambientales no han sido bien definidos y su rol es una incógnita. La influencia de aspectos nutricionales y eventuales virus en la génesis de la enfermedad no ha sido estudiada ${ }^{7}$.

\section{¿Por qué son LADA y no diabéticos tipo $1 ?$}

Para explicar la presentación más tardía y menos agresiva, se han planteado diferentes teorías. Pozilli y Di Mario postulan:

- Exposición menos marcada a factores ambientales.

- Menores títulos de anticuerpos específicos.

- Crisis intermitente de agresión autoinmune.

- Mayor capacidad para regenerar células beta y protección frente el proceso apoptótico.

- Inmunotolerancia adquirida.

Los últimos 3 puntos serían producto de un mejor balance genético protección/riesgo en comparación con diabéticos tipo $1^{1,13}$.

\section{Aspectos clínicos}

El diagnóstico se basa en 3 criterios que se enumeran en la Tabla 1.

\section{Edad de diagnóstico}

Si bien se define como característico de la enfermedad la aparición después de los 35 años, se describen casos a partir de los 25 años.

\section{Cuadro clínico}

En el estudio de Andersen, previamente mencionado, se encuentra que los pacientes portadores de LADA presentan mayor índice de masa corporal (IMC) que los diabéticos tipo 1, pero menor que los tipo 2. La condición de normopeso es el estado nutricional más frecuente. Es común la existencia de enfermedad autoinmune, o bien los antecedentes familiares de esta. La respuesta inicial satisfactoria a los hipoglicemiantes orales diferencia a estos pacientes de aquellos con DM tipo 1 tardía. La progresión hacia la insulinode-

Tabla 1. Criterios diagnósticos LADA

1. Aparición en la edad adulta, generalmente después de los 35 años

2. Presencia de auto anticuerpos específicos, siendo antiGAD el más prevalente

3. Sin necesidad de insulinoterapia al debut de la enfermedad, lo que debe prolongarse a lo menos por 6 meses 
pendencia es variable, desde 6 meses hasta varios años, dependiendo principalmente de los títulos de anticuerpos.

\section{Función beta pancreática}

En comparación con diabéticos tipo 2, presentan niveles de péptido $C$ reducido en ayuna y post estímulo ${ }^{14}$, aunque estos son mayores que pacientes con DM tipo 1 clásica o de aparición tardía, y su deterioro más lento en el tiempo. Al igual que en DM tipo 1 aquellos pacientes con edad de aparición más temprana y mayor número y títulos de anticuerpos específicos presentan un mayor y más rápido compromiso de la secreción insulínica ${ }^{2,15}$. Recientemente también se ha correlacionado el genotipo HLA DRB1-DQB1 con mayor propensión a la insulinodependencia ${ }^{16}$.

\section{Síndrome metabólico}

Dada la edad de aparición y la frecuencia de este síndrome en la población adulta, la coexistencia de ambas patologías no es infrecuente. Se calcula que aproximadamente $42 \%$ de los pacientes pueden presentar síndrome metabólico, cifra menor que en la DM tipo 2, en que la asociación llega al $84 \%{ }^{17}$. Es probable entonces que algunos pacientes presenten insulinoresistencia (IR) aunque la importancia de este fenómeno en la aparición de la enfermedad no está clarificado. Los niveles de adiponectina son similares a individuos sin DM, lo que sugiere que la IR no forma parte de la etiopatogenia de la enfermedad ${ }^{18}$. La presencia de dislipidemia e hipertensión arterial es mayor que en diabéticos tipo $1^{19}$, pero menos frecuente que en diabéticos tipo 2, lo que podría traducirse en un riesgo cardiovascular intermedio entre ambos tipos.

\section{Complicaciones crónicas}

Pocos estudios han abordado este tema. Cabrera-Rode et al. describen una menor incidencia de retinopatía, nefropatía y enfermedad vascular periférica, en comparación con diabéticos tipo 2 , aunque sin diferencias significativas dado el número reducido de pacientes. Recientemente, un estudio en Corea, con más de 300 pacientes (5,3\% clasificados como LADA, 70\% en insulinoterapia), revela que el riesgo de desarrollar complicaciones micro vasculares es similar a pacientes diabéticos tipo 1 y $2^{20,21}$.

\section{¿Cuándo pensar en la patología?}

Las características más frecuentemente asociadas a la enfermedad en comparación con diabéticos tipo 2 son: edad de inicio $<50$ años, síntomas agudos al debut, IMC $<25 \mathrm{~kg} / \mathrm{m}^{2}$, e historia personal o familiar de enfermedad autoinmune. La presencia de 2 o más de estos criterios presenta $90 \%$ de sensibilidad y $71 \%$ de especificidad para la identificación de pacientes LADA ${ }^{22,23}$.

En caso de sospecha deben solicitarse los anticuerpos específicos para confirmar el diagnóstico.

En la Tabla 2 se presentan algunas características diferenciales entre DM tipo 1, LADA y DM tipo 2.

\section{Tratamiento}

Hasta ahora no hay estrategias definidas con respecto al mejor tratamiento para estos pacientes. Los objetivos de control metabólico deben ser los recomendados para la enfermedad (HbAlc $\leq 7 \%)$. Las medidas dietéticas y ejercicio son similares a otros tipos de DM.

\section{Hipoglicemiantes orales}

- Sulfonilureas: son efectivas como reductoras de la glicemia, sin embargo, existen evidencias experimentales de que pueden aumentar la respuesta inmunológica, por lo que son con-

Tabla 2. Características de DM tipo 1, LADA y DM tipo 2

\begin{tabular}{|llll|}
\hline Características & DM tipo 1 & LADA & DM tipo 2 \\
\hline Edad de inicio & Infancia a adultez & Adulto & Adulto \\
\hline Síndrome metabólico & Similar población general & Similar población general & 80 a $90 \%$ \\
\hline Cetoacidosis & Frecuente & Infrecuente & Ausente \\
Autoinmunidad & Presente (predomina ICA) & Presente (predomina anti GAD) & Ausente \\
Insulinoterapia & Desde el diagnóstico & 6 meses sin requerimientos & Tardía \\
\hline
\end{tabular}


sideradas poco recomendables ya que podrían acelerar la progresión hacia la insulinodependencia $^{1,24}$.

- Metformina: su papel es poco claro en este tipo de patología. No existe evidencia de que proteja a la célula beta del deterioro secretor. Podría ser recomendable en casos asociados a IR o síndrome metabólico. En pacientes con DM tipo 1, un metanálisis reciente demuestra que su asociación a insulinoterapia sólo reduce los requerimientos de esta, sin beneficio en control metabólico o peso corporal ${ }^{7,25}$, por lo que su utilidad es discutible.

- Glitazonas (tiazolidinedionas): por su efecto antiinflamatorio y de protección de la apoptosis a la célula beta su uso parece recomendable en LADA. Resultados en modelos experimentales de DM autoinmune avalan este concepto y un estudio piloto en 23 pacientes sugiere que rosiglitazona añadido a insulina puede preservar la función de la célula beta ${ }^{26}$. Sin embargo, debe recordarse que la potencia hipoglicemiante de estas drogas es moderada y que su uso concomitante con insulina puede aumentar los efectos adversos (principalmente edema y retención hídrica) por lo que puede ser usada sólo en etapas tempranas de la enfermedad o bien para estabilizar la reserva pancreática. En un estudio reciente en China, el uso de rosiglitazona se asoció a un mejor control metabólico y preservación de péptido $C$ comparado con sulfonilureas, y a una preservación de la función beta al asociarse a insulina, en comparación con placebo ${ }^{27}$.

- Incretino-miméticos: si bien no existen experiencias con análogos de Glucagon Like Peptide-1 (GLP-1) o inhibidores de DPP-IV (gliptinas), su efecto antiapoptótico los convierten en una alternativa a futuro. Sin embargo, en un estudio reciente el uso de exenatide asociado a insulina en diabéticos tipo 1 no logró preservación del remanente de secreción pancreática ${ }^{28}$.

\section{Insulina}

Parece ser la terapia de elección, sobretodo en casos de control metabólico insuficiente. Al igual que en DM tipo 1, el esquema basal/bolus debiese ser considerado desde el inicio, aunque dada la estabilidad inicial de la enfermedad algunos pacientes pudiesen ser controlados con esquemas más sencillos (insulina basal o bifásica). Recientemente
Thunader et al demostraron que el tratamiento insulínico precoz es más efectivo en mantener un control metabólico óptimo (definido como HbAlc $\leq 7 \%$ ), después de 3 años, en comparación con hipoglicemiantes orales; sin embargo, no hubo diferencias entre ambos grupos con respecto a la conservación de la función beta pancreática ${ }^{29}$.

Debe mencionarse que un estudio aislado muestra que la adición de vitamina $\mathrm{D}(1-\alpha$-hidroxi D3) podría colaborar a preservar la reserva pancreática en pacientes con LADA, en asociación a la insulinoterapia ${ }^{30}$.

Finalmente, un metanálisis sobre tratamiento farmacológico ${ }^{24}$, con un total de 8 publicaciones (735 pacientes), concluye que:

- No hay beneficios en el control metabólico al asociar hipoglicemiantes a insulinoterapia.

- Mejor control metabólico con insulina en comparación a sulfonilureas.

- Insulinodependencia más temprana en pacientes tratados con sulfonilureas.

- Preservación de péptido C inicial con insulinoterapia precoz o rosiglitazona, lo que posicionaría a estas terapias como de elección.

Basado en esos antecedentes proponemos un algoritmo para el tratamiento (en casos de disponibilidad de glitazonas), considerando el nivel de HbAlc al inicio de la enfermedad (Figura 1).

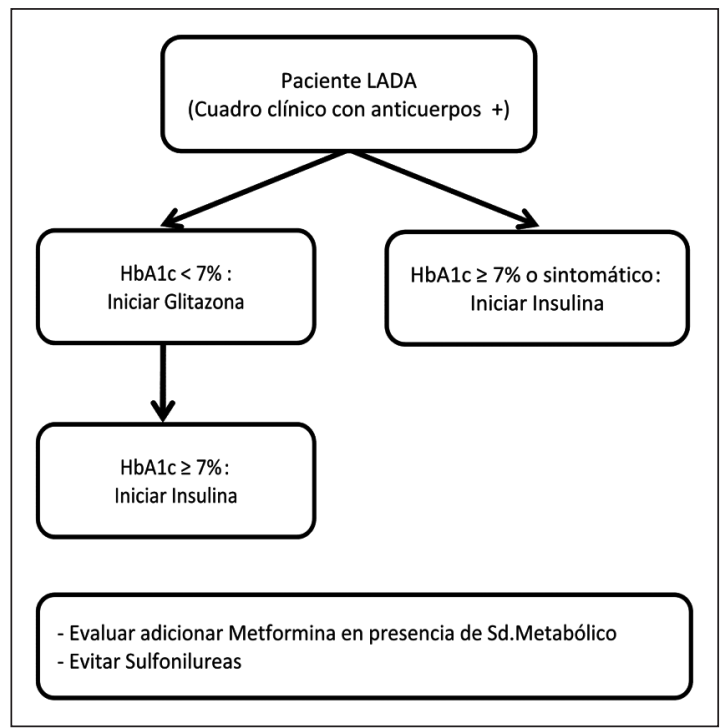

Figura 1. Algoritmo tratamiento. 


\section{Conclusiones}

La DM tipo LADA es una patología que podría ser considerada como la expresión menos agresiva de una enfermedad autoinmune, donde destacan los diabéticos tipo 1 clásicos y luego los de aparición tardía. Debe ser considerada en pacientes que se presenten como diabéticos tipo 2 "atípicos". Los factores más frecuentemente asociados a su presencia son: edad de aparición antes de los 50 años, estado nutricional normal e historia personal o familiar de autoinmunidad. El diagnóstico de certeza se hace a través de la detección de anticuerpos específicos, siendo el anti-GAD el de mayor prevalencia. La no requirencia de insulina al diagnóstico los diferencia de los caso de DM tipo 1 tardía. En nuestra opinión, las glitazonas, en etapas tempranas, y la insulinoterapia deben ser consideradas como las terapias de elección. Las sulfonilureas parecen ser perjudiciales sobre el deterioro de la reserva pancreática y debiesen ser evitadas, lo que acentúa la importancia de un diagnóstico precoz. Son necesarios más estudios para establecer la etiopatogenia, evolución y mejores alternativas terapéuticas en este tipo de DM.

\section{Referencias}

1. Pozilli P, Di Mario H. Autoimmune Diabetes not requiring insulin at diagnosis (latent autoimmune diabetes of the adult). Diabetes Care 2001; 24: 1460-7.

2. Stenstrom G, Gottsa A, Bakhtadze E, Berger B, Sundkvist G. Latent Autoimmune Diabetes in Adults: Definition, Prevalence, Cell Function, and Treatment. Diabetes 2005; 54 (2): 68-72.

3. Fourlanos S, Dotta F, Greenbaum C, Palmer J, Rolandsson $\mathrm{O}$, Colman P, et al. Latent autoimmune diabetes in adults (LADA) should be less latent. Diabetologia 2005; 48: 2206-12.

4. Ola T, Gigante A, Leslie R. Latent autoimmune diabetes of adults (LADA). Nutr Metab Cardiovac Dis. 2006; 16: 163-7.

5. Xiuying $\mathrm{Q}$, Jing S, Jing W Peizhong B Zhongliang X Madonna M, et al. Prevalence and correlates of latent autoimmune diabetes in adults in Tianjin, China: a population based cross-sectional study. Diabetes Care 2011; 34: 66-70.

6. Turner R, Stratton I, Horton V, Manley S, Zimmet P, Mackay I, et al. UKPDS 25: autoantibodies to islet-cell cytoplasm and glutamic acid decarboxylase for pre- diction of insulin requirement in type 2 diabetes: UK Prospective Diabetes Study Group. Lancet 1997; 350: 1288-93.

7. Leslie R, Williams R, Pozzilli P. Clinical Review: Type 1 Diabetes and Latent Autoimmune Diabetes in Adults: One End of the Rainbow. J Clin Endocrinol Metab 2006; 91 (5): 1654-9.

8. Hosszufalusi N, Vatay A, Rajczy K, Prohaszka Z, Pozsonyi E, Horvath L. Similar Genetic Features and Different Islet Cell Autoantibody Pattern of Latent Autoimmune Diabetes in Adults (LADA) Compared With Adult-Onset Type 1 Diabetes With Rapid Progression. Diabetes Care 2003; 26: 452-7.

9. Van Deutekom A, Heine R, Siemsek S. The islet autoantibody titres: their clinical relevance in latent autoimmune diabetes in adults (LADA) and the classification of diabetes mellitus. Diabet Med 2008; 25: 117-25.

10. Palmer J, Hampe C, Chiu H, Goel A, Brooks-Worrell B. Is Latent Autoimmune Diabetes in Adults Distinct From Type 1 Diabetes or Just Type 1 Diabetes at an Older Age? Diabetes 2005, 54 (2): 62-7.

11. Akesson Q, Uvebrant $\mathrm{K}$ Oderup Q, Lynch K, Harris RA Lernmark A et al. Altered natural killer (NK) cell frequency and phenotype in latent autoimmune diabetes in adults (LADA) prior to insulin deficiency. Clin Exp Immunol 2010; 161 (1): 48-56.

12. Andersen M, Lundgren V, Turunem J, Forsblom C, Isomaa B, Groop P, et al. Latent autoimmune diabetes in adults differs genetically from classical type 1 diabetes diagnosed after the age of 35 years. Diabetes Care 2010; 33 (9): 2064-10

13. Naik R, Palmer J. Latent Autoimmune Diabetes in adults. Rev Endocr Metab Disord 2003; 4: 233-41.

14. Yang L, Zhou Z, Huang G, Ouyang L, Li X, Yan X. Six year follow up of pancreatic B cell function in adults with latent autoimmune diabetes. World J Gastroenterol 2005; 11 (19): 2900-5.

15. Chaillous L, Bouhanick B, Kerlan V, Mathieu E, Lecomte $\mathrm{P}$, Ducluzeau $\mathrm{P}$, et al. Clinical and metabolic characteristics of patients with latent autoimmune diabetes in adults (LADA): absence of rapid beta-cell loss in patients with tight metabolic control. Diabetes Metab 2010; 36 (1): 64-70.

16. Maioli M, PesG, Delitala G, Puddu L, Falorni A, Tolu F. Number of auto antibodies and HLA genotype, more than high titers of glutamic acid decarboxylase auto antibodies, predict insulin dependence in latent autoimmune diabetes of adults. Eur J Endocrinol 2010; 163 (4): 541-9.

17. Hawa M, Thivolet C, Mauricio D, Alemanno I, Ciponneri E, Collier D, et al. Metabolic Syndrome and Autoim- 
mune Diabetes: Action LADA 3. Diabetes Care 2009; 32: 160-4.

18. Brophy S, Davies H, Stephens J, Prior S, Atkinson M, Bain S, et al. Adiponectin levels in people with Latent Autoimmune Diabetes-a case control study. BMC Res Notes 2010; 3: 317.

19. Weslley H Sepúlveda Amim R, Alvarenga T, Calsolari $\mathrm{M}$, Cavalcanti S. Comparison of Clinical and Laboratory Characteristics Between Adult-Onset Type 1 Diabetes and Latent Autoimmune Diabetes in Adults. Diabetes Care 2005; 28 (7): 1803-4.

20. Mi-Oh Roh, Chan-Hee Jung, Bo-Yeon Kim, Ji-Oh Mok, Chul-Hee Kim. The prevalence and characteristics of latent autoimmune diabetes in adults (LADA) and its relation with chronic complications in a clinical department of a university hospital in Korea. Acta Diabetol 2010 Oct 16 (online).

21. Isomaa $\mathrm{B}$, Almgren $\mathrm{P}$, Henricsson $\mathrm{M}$, Taskinen $\mathrm{M}$, Toumi T, Groop L, et al. Chronic Complications in Patients With Slowly Progressing Autoimmune Type 1 Diabetes (LADA). Diabetes Care 1999; 22 (8): 1347-53.

22. Fourlanos S, Perry C, Stein M, Stankovich J, Harrison L, Colman P. A Clinical Screening Tool Identifies Autoimmune Diabetes in Adults. Diabetes Care 2006; 29: 970-5.

23. Lutgens M, Meijer M, Peeters B, Poulsen M, Rutten M, Bots $\mathrm{M}$, et al. Easily obtainable clinical features increase the diagnostic accuracy for latent autoimmune diabetes in adults: An evidence-based report. Prim Care Diabetes. 2008; 2 (4): 207-11.
24. Brophy S, Brunt H, Davies H, Mannan S, Williams R. Interventions for latent autoimmune diabetes (LADA) in adults. Cochrane Database Syst Rev 2007; 18 (3): CD006165.

25. Abdelghaffar \$, Attia A. Metformin added to insulin therapy for type 1 diabetes mellitus in adolescents. Cochrane Database Syst Review 2009, 21 (1): CD006691.

26. Zhou Z, Li X, Huang G, Peng J, Yang L, Yan X, et al. Rosiglitazone combined with insulin preserves islet $\mathrm{B}$ cell function in adult onset latent autoimmune diabetes. Diabetes Metab Res Rev 2005; 21: 203-8.

27. Yang Z, Zhou Z, Li X, Huang G, Lin J. Rosiglitazone preserves islet $\mathrm{b}$-cell function of adult-onset latent autoimmune diabetes in 3 years follow-up study. Diabetes Res Clin Pract 2009; 83: 54-60.

28. Rother K, Spain L, Wesley R, Digon B, Baron A, Chen $\mathrm{K}$, et al. Effects of Exenatide alone and in combination with Daclizumab on B-cel function in long-stading type 1 diabetes. Diabetes Care 2009; 32: 2251-7.

29. Thunander M, Thorgeirsson H, Torn C, Petesrson C, Landin-Olsson M. B-cell function and metabolic control in latent autoimmune diabetes in adults with early insulin versus conventional treatment: a 3-year follow-up. Eur J Endocrinol 2011; 164: 239-45.

30. Li X, Liao I Yan X, Huang G, Lin, Lei M et al. Protective effects of 1-alpha-hydroxyvitamin D3 on residual beta-cell function in patients with adult-onset latent autoimmune diabetes (LADA). Diabetes Metab Res Rev 2009; 25 (5): 411-6. 